\title{
Ein Vorläufer Kants?
}

Rezension zu: Johann Nicolaus Tetens, Metaphysik, hrsg. von Michael Sellhoff, Hamburg: Meiner 2015.

Henning Tegtmeyer, Leuven

Als Philosoph ist Johann Nicolaus Tetens (1736-1807) dem philosophiehistorisch interessierten Publikum vor allem als möglicher Vorläufer Kants bekannt. Seit Hamann 1779 in einem Brief an Herder schrieb, dass Kant bei der Arbeit an der Kritik der reinen Vernunft den Tetens immer vor sich liegen habe, ${ }^{1}$ gilt das einerseits als ausgemacht. Andererseits ist in der Forschung noch immer umstritten, im welchem Sinne genau Tetens der Vernunftkritik und Transzendentalphilosophie Kants den Weg gebahnt haben soll. Dies hängt nicht nur mit der sich immer weiter ausdifferenzierenden Entwicklung von Lesarten der Kantschen Philosophie zusammen, sondern auch mit Schwierigkeiten, das Denken Tetens ‘ in den Zusammenhang der deutschen Philosophie des 18. Jahrhunderts einzuordnen. Denn einerseits gehört Tetens klarerweise in die Tradition der als rationalistisch geltenden Wolffschen Schulphilosophie, andererseits gilt er, vor allem in seiner Psychologie, als Empirist, gar als ,deutscher Locke‘. ${ }^{2}$ Das lässt ihn zum einen wie einen Brückenbauer zwischen Rationalismus und Empirismus aussehen. Kant selbst nimmt ja in der Methodenlehre der Kritik der reinen Vernunft für sich in Anspruch, eine Synthese zwischen diesen beiden gegensätzlichen Hauptströmungen der Philosophie geleistet zu haben. Zum anderen scheint ihn die Nähe sowohl zu Wolff als auch zu Locke aber gerade von Kant wegzurücken, betont dieser doch den prinzipiellen Unterschied zwischen seiner eigenen, transzendentalen Analyse der Bedingungen menschlicher Erkenntnis und der bloßen ,Physiologie‘ menschlicher Erkenntnis bei Locke und dessen Schülern einerseits, einer bloß dogmatischen Metaphysik ohne vorausgehende Kritik andererseits.

Zur Klärung dieser Problematik trägt die nun erstmals veröffentlichte Metaphysik-Vorlesung Tetens' insofern bei, als sie zeigt, auf welche Weise dieser versucht, metaphysische Theoreme der Wolffschen Philosophie auf eine erkenntnistheoretische, zum Teil stark an empiristischen Vorbildern orientierte Grundlage zu stellen. Das ist im Zusammenhang des Wolffianismus durchaus nichts Außergewöhnliches, wie der Herausgeber in seiner umfangreichen und sachkundigen Einleitung verdeutlicht. Denn die rationalistischen und die empiristischen Tendenzen der deutschen Aufklärungsphilosophie nach 1750 gehen großenteils aus der Schule Wolffs hervor und gründen sich letztlich in Wolffs eigenem Streben nach einer empirischen

\footnotetext{
${ }^{1}$ Vgl. die Einleitung des Herausgebers, Anm. 22.

${ }^{2}$ Vgl. Marta Zappalorto, Johann Nicolaus Tetens - Il Locke tedesco?, Soveria Mannelli: Rubbettino 2011.
} 
Absicherung seines philosophischen Systems. ${ }^{3}$ Dass schon Wolff selbst und nicht erst Kant nach einer Synthese rationalistischer und empiristischer Denkweisen und Forschungsmethoden strebt, stützt eine Tendenz der neueren philosophiehistorischen Forschung, den analytischen Wert der Empirismus-Rationalismus-Dichotomie für das Verständnis der Philosophie des 17. und 18. Jahrhunderts insgesamt in Frage zu stellen. ${ }^{4}$

Darüber hinaus kann diese Publikation aus sich heraus aber nicht erhellen, auf welche Weise Tetens zur Formung von Kants Projekt beigetragen haben könnte, und zwar aus dem einfachen Grund, dass der Text auf einer in Reinschrift ausgeführten Nachschrift einer im Wintersemester 1788/89 in Kiel gehaltenen Metaphysikvorlesung beruht. Diese Vorlesung wurde also erst nach dem Erscheinen sowohl der ersten als auch der stark überarbeiteten zweiten Auflage der Kritik der reinen Vernunft gehalten, und Kant wird in ihr mehrfach erwähnt. Wenn man also die Einflussfrage stellen will, dann darf hier gefragt werden, inwiefern hier umgekehrt von einem Einfluss Kants auf Tetens die Rede sein kann. Den Wert dieser Publikation schmälert das aber nicht im geringsten; im Gegenteil: die Lektüre der Tetensschen Metaphysik regt zu Fragen an, die in der bisherigen Forschung kaum thematisiert wurden, vor allem im Hinblick auf die Kontinuität zwischen der deutschen Schulphilosophie des 18. Jahrhunderts einerseits und dem Deutschen Idealismus andererseits, und zwar über die tatsächliche oder vermeintliche Zäsur der Kantschen Transzendentalphilosophie hinweg. Dazu im Folgenden mehr.

Wenn nun im Hinblick auf Kant etwas an Tetens، reifem System der Metaphysik auffällt, dann ist es die weitgehende Abwesenheit zentraler Kantscher Denkmotive, und das trotz der hohen Wertschätzung, die Tetens für Kant zum Ausdruck bringt. Weder macht sich Tetens das Anliegen einer Begrenzung der Ansprüche des rein spekulativen Denkens zu eigen, um so Platz für das praktische Denken und den Glauben zu machen, noch zeigt er sich beunruhigt von den Antinomien, in die sich reines, nicht durch Erfahrung begrenztes metaphysisches Denken verstricken kann oder gar muss, wie Kant meint. Auch teilt er nicht wie Kant Humes Zweifel an der Legitimität der Begriffe der Substanz und der Kausalität. Was hinsichtlich der Motivation zur Metaphysik allenfalls an Kant erinnert, ist die Unterscheidung zwischen einer ,natürlichen Metaphysik`, die auf den Allgemeinbegriffen beruht, welche „den gemeinen Verstand (sensus communis)“ ausmachen, und einer durch Wissenschaft verdeutlichten, geprüften und theoretisch geordneten Metaphysik, die zu formulieren Aufgabe der Philosophie ist. ${ }^{5}$

\footnotetext{
${ }^{3}$ Vgl. Einleitung, S. XX1 f.

${ }^{4}$ Vgl. Werner Schneiders (Hg.), Christian Wolff 1679-1754. Interpretationen zu seiner Philosophie und deren Wirkung, Hamburg: Meiner 1983; Hans-Jürgen Engfer, Empirismus versus Rationalismus? Kritik eines philosophiegeschichtlichen Schemas, Paderborn: Schöningh 1996.

5 Tetens, Metaphysik, Vorbericht, § 4, S. 4 f., Hervorhebung i. Orig.
} 
Tetens erwähnt in diesem Zusammenhang neben der ersten Kritik auch die Prolegomena Kants ausdrücklich. ${ }^{6}$ Die kritische Terminologie der Kantschen Transzendentalphilosophie bleibt dagegen weitgehend ohne Echo bei Tetens. Dagegen macht er wie Kant reichlich Gebrauch von der scholastischen Terminologie der Wolffschen Schulphilosophie. Terminologische Parallelen zwischen Wolff, Baumgarten, Tetens und Kant werden im ebenfalls umfangreichen Anmerkungsapparat minutiös ausgewiesen.

Tetens präsentiert jedoch seine eigene, von Wolff und Baumgarten im Aufbau zum Teil stark abweichende Entwicklung eines metaphysischen Systems. Wenn man die Differenz zwischen diesem Projekt und dem Kants beschreiben wollte, dann müsste man wohl sagen, dass es Tetens im Unterschied zu Kant nicht so sehr um eine kritische Begrenzung als vielmehr um eine erkenntnistheoretische Grundlegung der Metaphysik geht. Deswegen kann er seine Metaphysik nicht wie Baumgarten mit der allgemeinen Ontologie beginnen, sondern schickt dieser eine gleichermaßen an Locke und Wolff angelehnte empirische Psychologie voraus. Für dieses ungewöhnliche, aber im Kontext Wolffschen Denkens durchaus begründbare Vorgehen führt Tetens selbst einen didaktischen Grund an, nämlich „um den Verstand zur Bearbeitung der Allgemeinbegriffe in der Ontologie vorzubereiten“. ${ }^{7}$ Das legt nahe, dass auch ein anderer Einstieg in die Metaphysik möglich wäre. Dagegen spricht der durchweg systematischsynthetische, von einfachen zu immer komplexeren und voraussetzungsreicheren Begriffen fortschreitende Duktus dieser Vorlesung, der einem systematischen Bauprinzip folgt. Demnach können und müssen die Begriffe und Prinzipien der allgemeinen Ontologie, der ,raisonnirenden“, also rationalen Psychologie, der rationalen Kosmologie und der philosophischen Theologie aus den Allgemeinbegriffen des Denkens überhaupt entwickelt werden. Diese werden aber nicht wie bei Kant als ein System wechselseitig voneinander abhängender reiner Verstandesbegriffe und darauf beruhender Verstandesprinzipien präsentiert, die dann durch Schematisierung in geeignete Beziehungen zu Anschauungen treten müssen, um Erkenntnisse zu ermöglichen. Bei Tetens werden die allgemeinen Denkbestimmungen vielmehr genetisch aus einer Analyse der Stufen unseres Bewusstseins von Objekten und der möglichen Relationen zwischen Bewusstsein und Bewusstseinsgegenstand gewonnen. Die Analyse ist ,empirisch', d.h. auf Selbstreflexion gegründet. Ganz in der Tradition Wolffs wird das Bewusstsein von etwas als potentiell graduell aufgefasst, wobei die Grade der Klarheit und Deutlichkeit der Akt- und Gegenstandserfassung die verschiedenen Bewusstseinsniveaus und die ihnen entsprechenden Akte selbst konstituieren. Im Zentrum steht dabei der Begriff der Vorstellung (repraesentatio), der von dem der Empfindnis (Affektion), also der emotionalen Färbung einer

\footnotetext{
${ }^{6}$ Vgl. ebd., § 5, S. 5 .

${ }^{7}$ Vgl. ebd., § 2, S. 4, Hervorhebung i. Orig.
} 
Vorstellung, strikt unterschieden wird. ${ }^{8}$ Die allgemeinen Unterschiede zwischen Fühlen, Wahrnehmen und Erkennen werden als graduelle gedeutet, ebenso wie im Felde des praktischen Bewusstseins die Unterschiede zwischen Begehren und Verabscheuen, Wollen und Nichtwollen mitsamt der dazu nötigen Seelenvermögen (Triebe). Hinsichtlich der Objekte der Vorstellungen werden einfache von komplexen Vorstellungen unterschieden, wobei darin schon Hinweise auf die innere Natur der vorgestellten Objekte und ihrer Gegebenheitsweise (z.B. als wirkliche oder fiktive, imaginierte oder wahrgenommene, etc.) enthalten sind. Aus dieser empirischen Selbstreflexion versucht Tetens auch schon erste Anhaltspunkte für die Thematisierung des Problems der Willensfreiheit und ihrer Vereinbarkeit mit dem Satz des zureichenden Grundes sowie für das Verhältnis von Leib und Seele zu gewinnen.

Auf Grundlage der empirischen Psychologie entwickelt Tetens seine allgemeine Ontologie als ein System der allgemeinen Verstandesbegriffe, die dabei in Wolffscher Manier als klare und deutliche Vorstellungen entwickelt werden. Auch dieses System wird genetisch als ein System fortschreitender Differenzierungen vorgestellt, die vom Möglichen und Unmöglichen (nach dem Satz des Widerspruchs) über Etwas und Nichts, Wirklichkeit, Identität und Differenz, Koexistenz und Dependenz, Bestimmung und Bestimmtes, Qualität, Quantität, Realität und Negation, Vollkommenheit, Ordnung und Güte zu den ontologischen Kernbegriffen von Substanz und Akzidenz, Raum und Zeit, Kausalität und Kraft führt. ${ }^{9}$ Dieser Hauptteil der Tetensschen Metaphysik kulminiert in den theologisch bedeutsamen Begriffen eines notwendigen Wesens (ens necessarium), das zugleich als Inbegriff aller Vollkommenheiten (omnitudo realitatis) und damit als metaphysisch unendlich gedeutet wird. Damit werden die Weichen für die metaphysische Theologie schon mitten in der allgemeinen Ontologie gestellt. Gleiches gilt für die Einführung des Begriffs der Erstursache, der für die Willensfreiheitsproblematik und für die Schöpfungstheologie gleichermaßen unverzichtbar ist.

Die Teile der speziellen Metaphysik selbst, die rationale Psychologie, Kosmologie und Theologie also, können vergleichsweise knapp vorgetragen werden, weil vieles bereits in der allgemeinen Ontologie vorentschieden ist. Tetens positioniert sich hier in den Fragen der Verbindung der Seele mit dem Leib und ihrer Unsterblichkeit (wobei er sich auf die Anhaltspunkte stützen kann, womit ihn die empirische Psychologie ausstattet), der Unendlichkeit und Vollkommenheit der Welt und letztlich des Wesens Gottes in sich und in Bezug auf die Welt. In der rationalen Psychologe führen ihn seine empirischen Betrachtungen zur transtemporalen Identität des Bewusstseins (der Seele) zur These von der metaphysischen Verschiedenheit

\footnotetext{
${ }^{8}$ Vgl. ebd., Psychologia empirica I, § 24, S. 20.

${ }^{9}$ Das Verfahren erinnert nicht ohne Grund an die Wissenschaftslehre Fichtes und die Wissenschaft der Logik Hegels. Ich werde auf derartige Parallelen zum Schluss zurückkommen.
} 
von Leib und Seele, die er mit Leibniz als Monade deutet, und folglich zur Zurückweisung des materialistischen Monismus. Hinsichtlich der substantiellen Verbindung zwischen Leib und Seele und den Ursachen dieser Verbindung unterscheidet er verschiedene rationalistische Hypothesen, ohne sich auf eine festzulegen. Dagegen hält er es für „moralisch gewiss“, dass unsere Seele nach dem Tod fortbesteht. Wie Kant argumentiert er in diesem Zusammenhang mit der Möglichkeit der fortschreitenden Perfektion der Seelenvermögen. Vereinige man diese empirisch beobachtete Möglichkeit mit dem metaphysischen Gedanken eines gütigen Gottes, so sei man berechtigt anzunehmen, dass dieser Perfektionsprozess mit dem physischen Tod nicht ende. ${ }^{10}$ In der rationalen Kosmologie argumentiert er zwar nicht für eine Pluralität möglicher Welten wie Leibniz, wohl aber für dessen These, dass die Welt bestmöglich sei, wobei die Welt als durchgehend geordneter Zusammenhang kontingent existierender einfacher Substanzen gedeutet wird. Damit wird zugleich ein Schluss auf eine intelligente und gütige Erstursache, einen Schöpfergott möglich, der den Kerngehalt der rationalen Theologie ausmacht. Auf dieser Grundlage werden Atheismus und Polytheismus zurückgewiesen. Da Tetens in seiner Kausaltheorie ferner die metaphysische Verschiedenheit von Ursache und Wirkung bewiesen hat, kann er hier auch die Identität von Gott und Welt und damit den Pantheismus und Spinozismus mit Gründen verwerfen. Das ist eine weitere Pointe seines kosmologischen Arguments für die Existenz eines personalen Gottes.

Dass auf diese Weise ein vollständiges, komplexes und durchdachtes System der Metaphysik formuliert wird, steht außer Zweifel. Durch die Publikation der Metaphysik-Vorlesung werden die Strukturen dieses Systems nun greifbar. Das kann allerdings nicht heißen, dass Tetens' Metaphysik ohne Schwierigkeit wäre. Hier sollen nur zwei ins Auge fallende Probleme benannt werden: (1) Anhänger Kants werden sich an dem Gedanken stoßen, dass Metaphysik, die Kant als apriorische Wissenschaft vor aller Erfahrung kennzeichnet, auf Erfahrung gründen sollte, und zwar nicht nur in ihrem empirischen Teil, sondern durchgehend. Für das genaue Verständnis dieser anscheinend ganz unkantischen Auffassung der Möglichkeit von Metaphysik hängt aber wohl alles davon ab, was genau unter Erfahrung eigentlich verstanden werden soll. Auffällig ist, dass Tetens sich in seinen Appellen an unsere Erfahrung nahezu ausschließlich auf die innere Erfahrung selbstreflexiver Akte bezieht. Das scheint ihn wegzurücken von einem Verständnis der inneren Erfahrung als Introspektion wie bei Locke und Hume und vielmehr vorauszuweisen auf eine Theorie selbstbewusster Akte, wie man sie dem Ansatz nach auch bei Kant, ausgeführt aber bei Fichte und dann wieder in der Phänomenologie Brentanos und Husserls findet. Wie groß man hier die methodische Differenz zwischen

\footnotetext{
${ }^{10}$ Vgl. Tetens, Metaphysik, Grundsätze der raisonnirenden Psychologie, § 23, S. 258.
} 
Kant und Tetens ansetzt, wird also maßgeblich von der Interpretation des Tetensschen Erfahrungsbegriffs abhängen. (2) Eigentümlich in der Schwebe bleibt der ontologische Status der den ontologischen Begriffen korrespondierenden Gegenstände. Handelt es sich dabei um Dinge in einer bewusstseinstranszendenten, an sich seienden Wirklichkeit, oder handelt es sich um bloße Vorstellungen? Tetens selbst neigt erkennbar realistischen Positionen zu, und ganz in diesem Sinne unterscheidet er durchgehend ein wahrgenommenes von einem bloß vorgestellten Ding, ein wirksames von einem bloß als wirksam vorgestellten Ding, eine Reihe von Ereignissen im Unterschied zu einer Reihe von Vorstellungen, etc. Diese Unterschiede kann er erläutern, indem er auf die, modern gesprochen, unterschiedlichen inferentiellen Verpflichtungen hinweist, die wir uns durch den Gebrauch des ontologisch jeweils stärkeren Begriffs eingehen. Und doch bleiben ein wirkliches Ding und die Vorstellung von einem wirklichen Ding zweierlei.

Ungeachtet derartiger und anderer Schwierigkeiten, mit denen sich die Tetens-Exegeten befassen werden, besteht ein großer Wert dieser Wiederentdeckung eines vermeintlich, vorkantischen' Philosophen darin, dass sie uns veranlassen kann, das vor allem von Kant selbst erzeugte und vom Neukantianismus kanonisierte Bild der deutschen Philosophie gründlich zu hinterfragen. Was in diesem Bild immer zu großen Teilen ein Rätsel bleiben musste, ist nicht so sehr die tiefe Unzufriedenheit des Deutschen Idealismus mit der Philosophie Kants überhaupt als vielmehr die ungeheuer gestiegenen Anforderungen an Systematizität und Methode, denen sich Reinhold, Fichte und Hegel unterwerfen, ebenso auf seine ganz eigene Weise auch Schelling. ${ }^{11}$ Dass aber ein System der Philosophie nur dann ein Ganzes sein kann, wenn es aus einem wahren und als gültig ausgewiesenen Grundprinzip entwickelt wird, ist kein Gedanke, den Reinhold, Fichte und ihre Nachfolger neu formulieren oder wozu sie eigens Spinoza hätten studieren müssen. Sie waren mit diesem Gedanken schlicht aus den schulphilosophischen Lehrbüchern vertraut, aus denen sie ihre philosophische Bildung zuerst bezogen hatten. Kenntnis der Wolffschen Philosophie war bis zu Hegel und Schelling und zum Teil darüber hinaus noch selbstverständliche Bildungsvoraussetzung des im (protestantischen) Deutschland tätigen akademischen Philosophen. Das anzuerkennen heißt nicht, die immense Bedeutung von Kants Denken als Anregung, Herausforderung und auch als Stein des Anstoßes für den Deutschen Idealismus abzustreiten. Es kann aber heißen, die Kant-Rezeption der nachfolgenden Generation viel selbstverständlicher vor dem Horizont übergreifender Konti-

\footnotetext{
${ }^{11}$ Vgl. Paul Franks, All or Nothing. Systematicity, Transcendental Arguments, and Skepticism in German Idealism, Cambridge, Mass.: Harvard University Press 2005. Franks sieht diesen Zug zur strengen Systematik vor allem als Antwort auf den postkantianischen Skeptizismus bei Schulze und Maimon, nicht aber als Anknüpfung an traditionellere Systemvorstellungen. Dem Einfluss Spinozas schreibt er allerdings eine Schlüsselrolle für die Genese und Entwicklung des Deutschen Idealismus zu.
} 
nuitäten im Philosophieverständnis zwischen Schulmetaphysik und idealistischer Spekulation zu sehen. Es war eben nicht nur Kant, sondern es waren auch und gerade die jungen Denker nach ihm, die sich von Wolffs Geist der Gründlichkeit und systematischen Strenge zutiefst angezogen fühlten. Um das neu zu sehen, bedarf es sicher nicht der Metaphysik Tetens' als solcher, der man wohl keinen Einfluss auf die Bildung der idealistischen nachkantischen Philosophie wird nachsagen können. Sehr wohl aber gehört sie in das volle Bild der langen Nachwirkung der Wolffschen Philosophie. Die Stellung Tetens‘ in dieser Geschichte vor und nach Kant ist nun deutlicher geworden. 